Supporting Information for

\title{
Tailoring the Electrode Interface with Enhanced Electron Transfer for High-Rate Lithium-Ion Battery Anodes
}

Junyi $\mathrm{Ji}^{a}$, Xinghong Cui ${ }^{a}$, Yanfang Zhu ${ }^{a}$, Linfei Lai ${ }^{c}$, Xin Zhao ${ }^{d}$, Yuxin Zhang ${ }^{e}$, Lili Zhang $^{b}$ *

${ }^{\mathrm{a} C o l l e g e}$ of Chemical Engineering, Sichuan University, Chengdu, Sichuan 610065, P. R. China

${ }^{\mathrm{b}}$ Institute of Chemical and Engineering Sciences, A*STAR, 1 Pesek Road, Jurong Island 627833, Singapore

${ }^{c}$ Key Laboratory of Flexible Electronics (KLOFE) \& Institute of Advanced Materials (IAM), Jiangsu National Synergistic Innovation Center for Advanced Materials (SIC AM), Nanjing Tech University, 30 South Puzhu Road, Nanjing 211816, P. R. China

${ }^{\mathrm{d}}$ College of Material Science \& Engineering, State Key Laboratory for Modification of Chemical Fibers and Polymer Materials, Donghua University, Shanghai 201620, P.

R. China

${ }^{\mathrm{e}}$ College of Material Science and Engineering, Chongqing University, Chongqing 400044, P. R. China

\section{*Corresponding author}

Lili Zhang, Email: zhang_lili@,ices.a-star.edu.sg 


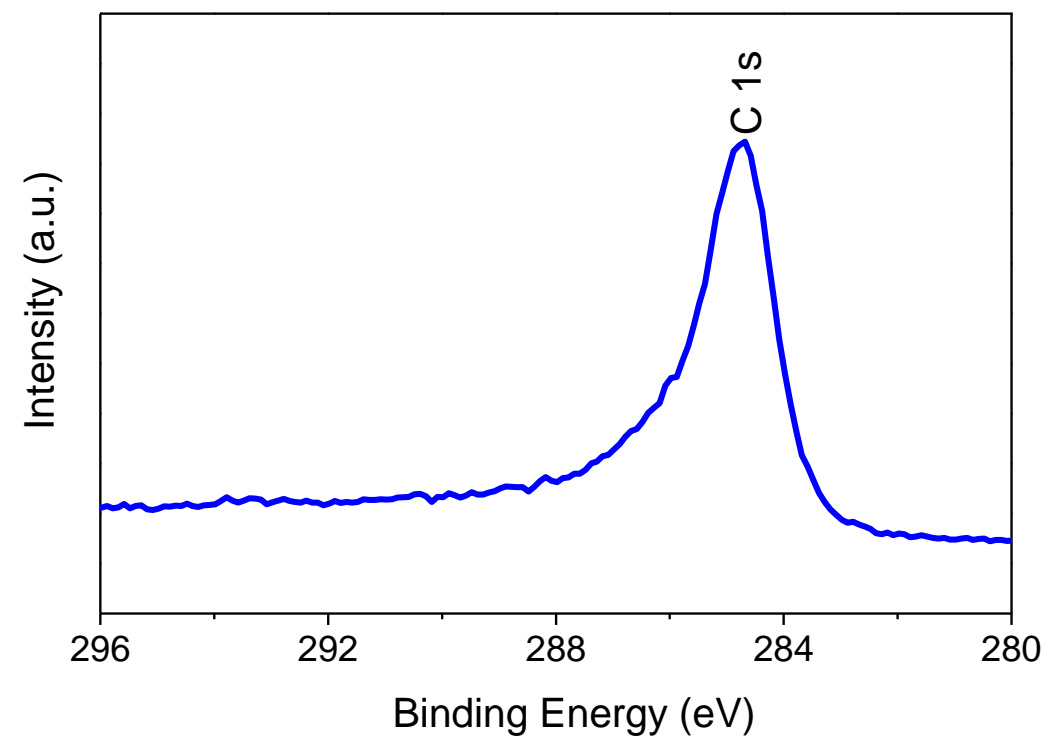

Figure S1. XPS C 1s spectrum of the aNGO/Ni.
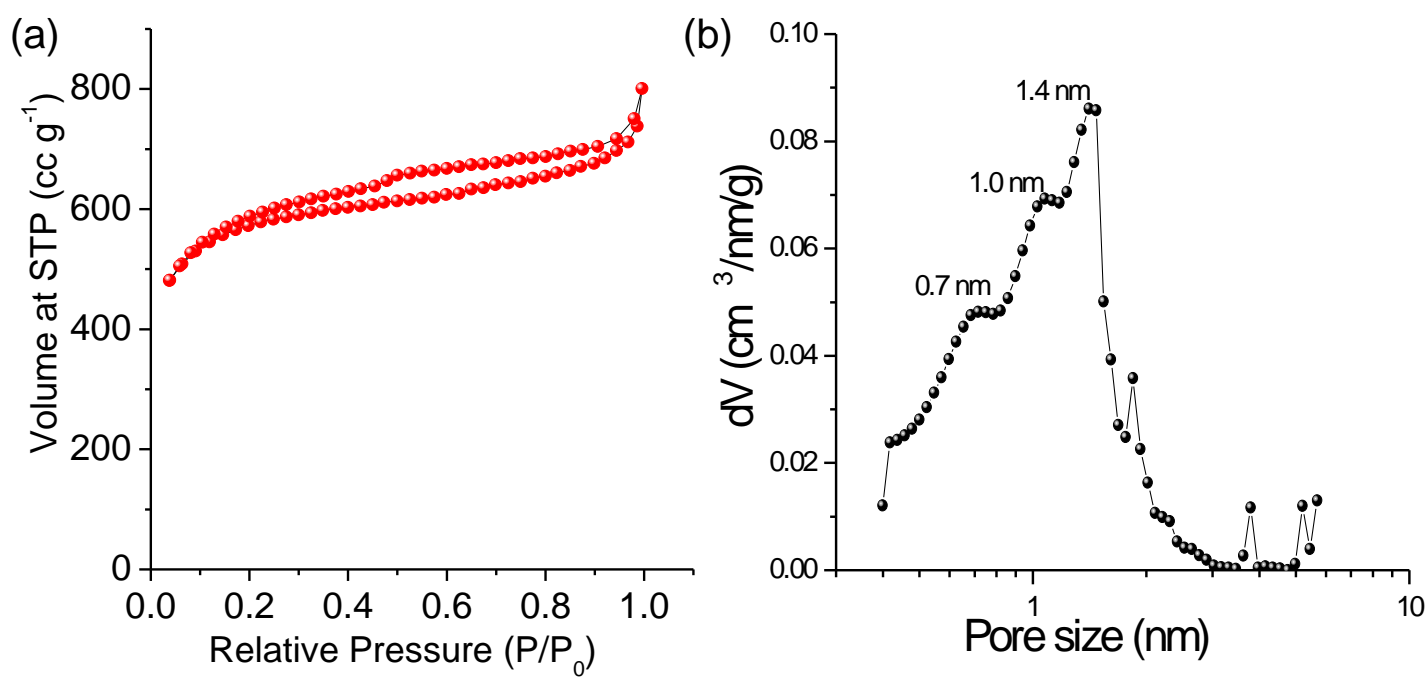

Figure S2. a) Nigrogen sorption and b) related pores distribution of the aNGO powder. 

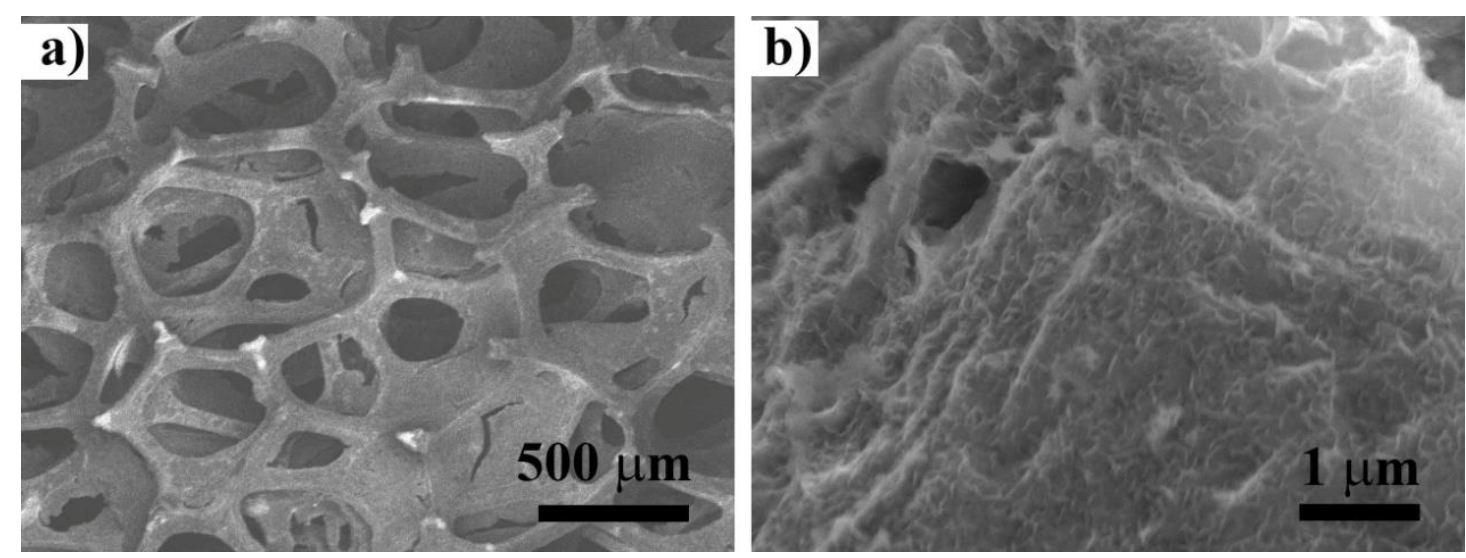

Figure S3. SEM images of a) aNGO film partially covered the Ni foam pores, and b) relatively smooth aNGO layers intimately covered on the Ni foam struts.
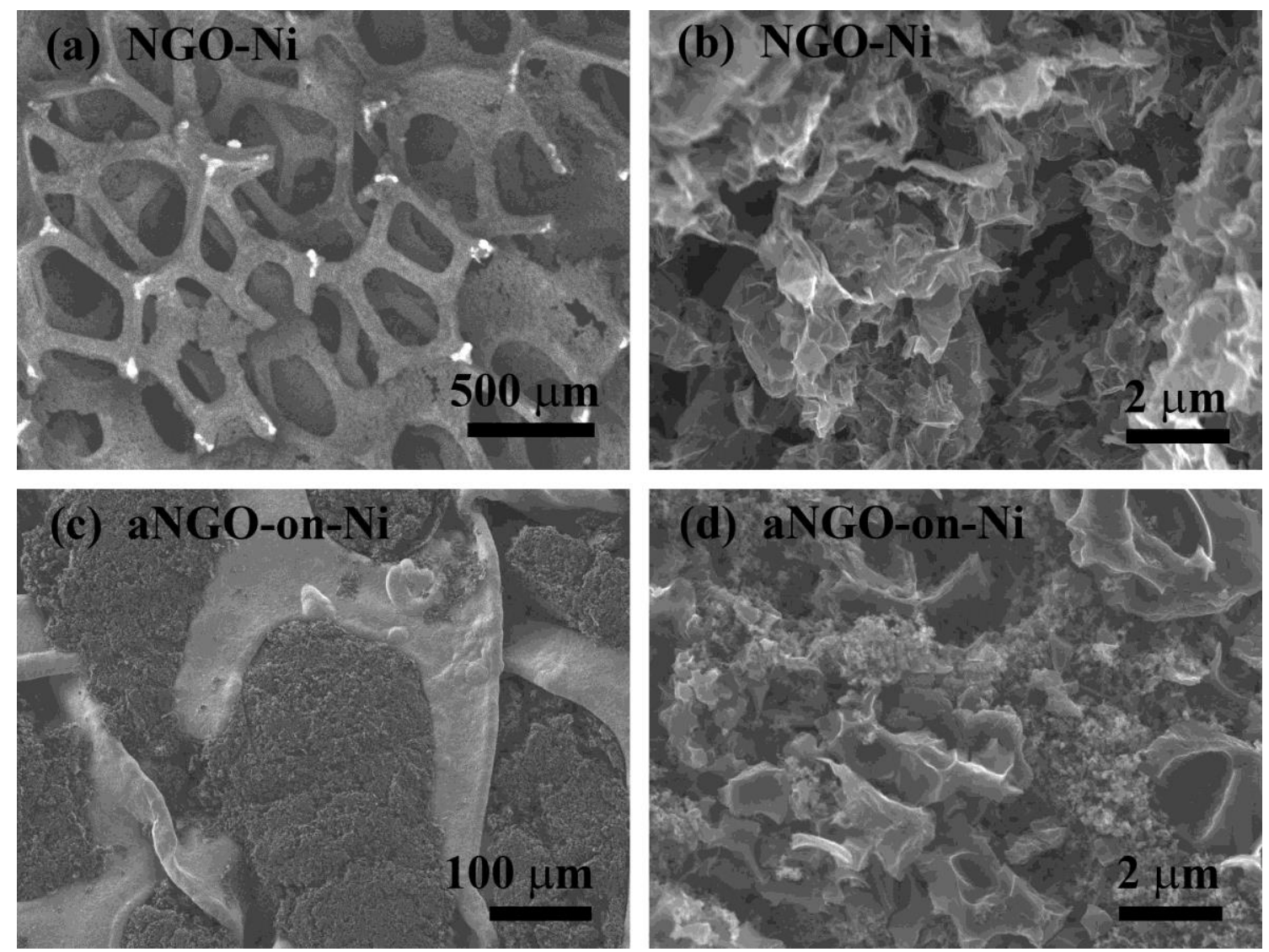

Figure S4. SEM images of the a, b) NGO-Ni and c, d) aNGO-on-Ni with discontinuous distribution and crumpled structure. 

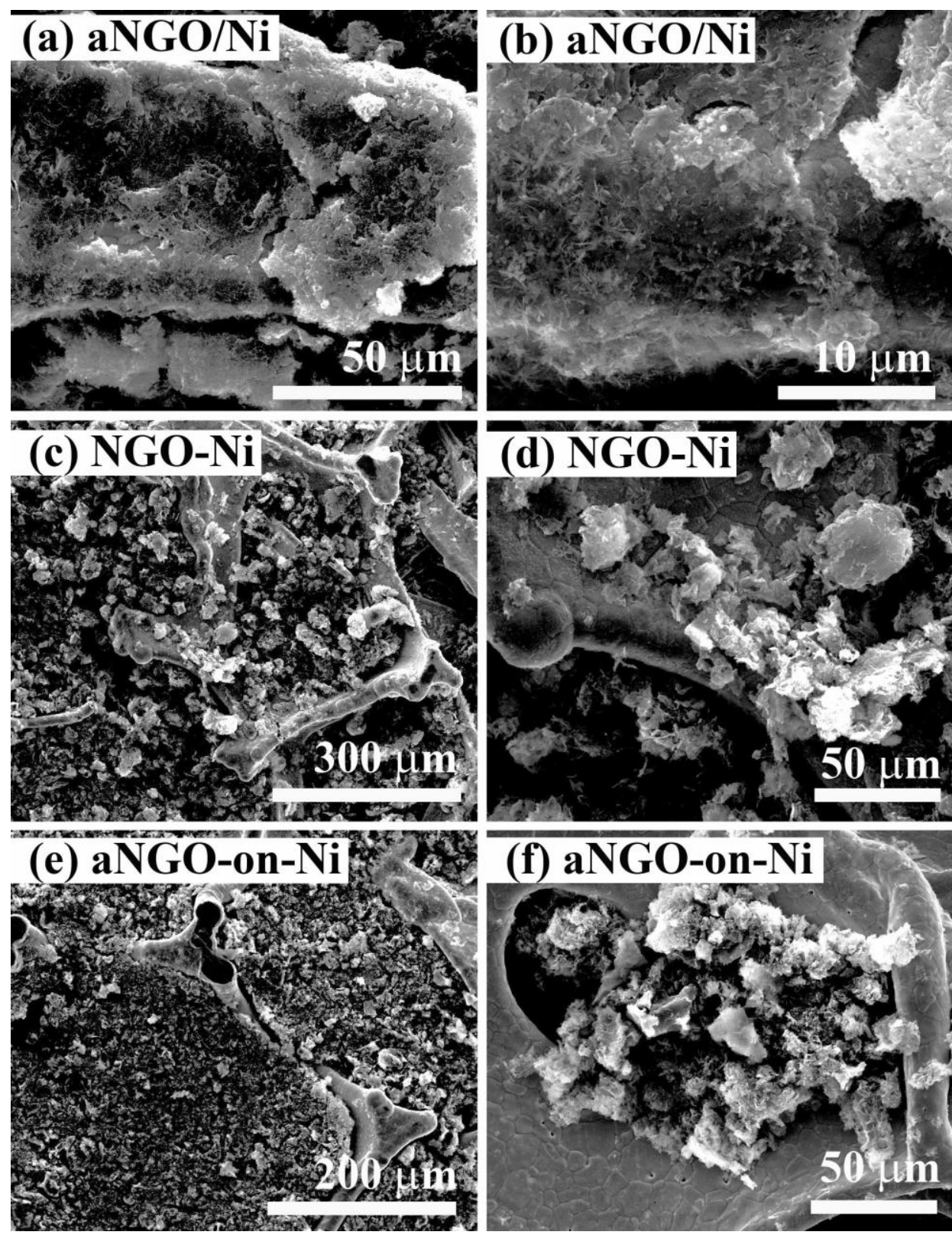

Figure S5. SEM images of the aNGO/Ni (a, b), NGO-Ni (c, d), aNGO-on-Ni (e, f) electrodes after long cycling test. 

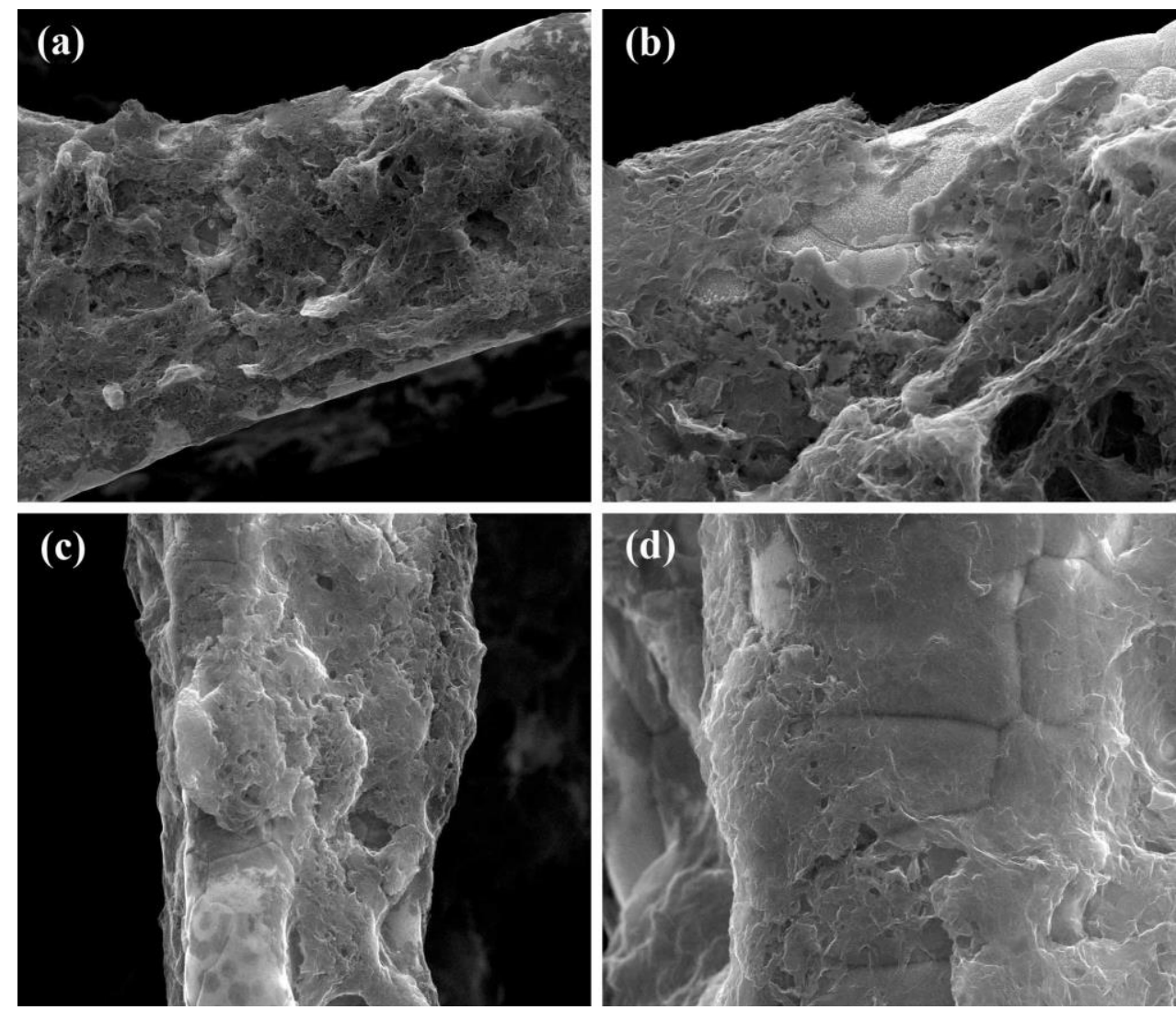

Figure S6. The amount of $\mathrm{KOH}$ can also influence the productivity and the morphology of the aNGO. The SEM images show the aNGO/Ni with a

KOH/GO@PPy ratio of 2:1 (a,b) and 4:1 (c,d), respectively. The sample with 2:1 ratio shows a relatively crumpled structure, and the contact between aNGO and nickel foam is not so tight (figure S6b). On the other hand, the sample with 4:1 ratio shows similar 'sheet contact'; the aNGO film tightly anchored on Ni foam struts can be seem from figure S6d. However, the yield of the sample with 4:1 ratio is very low (50-70\% for 3:1 and $20-30 \%$ for 4:1). Therefore, the KOH/GO@PPy ratio of 3:1 is adopted in this work. 